\title{
SIMULADOR DE UMA REDE CELULAR BASEADA NO SISTEMA FDMA (AMPS/TDMA)
}

\author{
AILTON AKIRA SHINODA ${ }^{1}$
}

SHINODA, A. A. Simulador de uma rede celular baseada no sistema FDMA (AMPS/TDMA). Semina: Ci. Exatas/Tecnol. Londrina, v. 21, n. 4, p. 47-51, dez. 2000.

RESUMO: Este artigo descreve o modelo e a implementação de um simulador de uma rede celular baseada no sistema FDMA (AMPS/TDMA). O simulador tern como objetivo a avaliação do desempenho da rede celular FDMA e a otimização de certos parâmetros. Alguns resultados obtidos do simulador, também são fornecidos.

PALAVRAS-CHAVE: rede celular, simulação, FDMA, AMPS, TDMA.

\section{INTRODUÇÃO}

Ferramentas de simulação que possibilitem aos pesquisadores, projetistas ou operadores uma avaliação correta do desempenho dos sistemas celulares, sobre uma grande gama de condições, estão se tornando cada vez mais importante (Woerner et al., 1994; Tranter \& Kosbar, 1994). Em sistemas de grande porte e completamente inviável a análise analítica do desempenho, levando em consideração os aspectos dinâmicos das interações envolvidas entre as ERB's e os usuários tais como o handoff, velocidade, tempo de duração da chamada, distribuição dos usuários na área de serviço, entre outros. Assim a simulação torna-se uma ferramenta essencial, tanto na avaliação da performance como na otimização dos sistemas celulares.

Este artigo descreve o modelo e implementação de um simulador de rede celular baseado no sistema FDMA (AMPS/TDMA). Em termos de alocação de canais o TDMA pode ser considerado como um caso particular do sistema AMPS (Mac Donald, 1980). Assim o simulador focaliza a avaliação da performance do sistema AMPS sobre várias condições de tráfego, dado um conjunto de parâmetros do sistema tais como número de canais por ERB, distância entre as ERB's, velocidade máxima/mínima. As principais saídas ou resultados são área de cobertura, topologia das ERB's, grau de serviço, distribuição dos handoffs, número total de chamadas e movimento do usuário durante a chamada.

O restante do artigo e organizado da seguinte forma. Na seção 2 é apresentado o sistema a ser modelado e simulado. Na seção 3 é descrito a ferramenta de simulação que foi utilizada para construir o simulador. $\mathrm{Na}$ seção 4 são apresentados alguns resultados do simulador. Finalmente, na seção 5 , a conclusão do artigo com algumas propostas para trabalhos futuros.

\section{SISTEMA}

Um sistema celular consiste basicamente de 4 entidades ou elementos:

- estações móveis;

- estações rádio base (ERB);

- centro de comutação e controle (CCC);

- ligações.

A estação móvel possui uma unidade de controle, um transceptor e uma antena.

A estação rádio base fornece uma interface entre as estações móveis e a CCC. A ERB possui uma unidade de controle, antenas, conexões de rádio e unidade de potência.

As ligações oferecem a conectividade entre as estações móveis, A CCC é o coração do sistema. Ela coordena e administra todas as ERB's. Além disso, a CCC interfaceia com a Rede Telefônica Pública Comutada (RTPC), controla o processamento da chamada e gerencia as atividades da tarifação. As CCC's comutam as chamadas entre as estações móveis ou com assinantes fixo da rede RTPC.ERB's e a CCC. Conexões de rádio trafegam informações entre as estações móveis e as ERB's. Conexões de microondas ou cabos estabelecem uma alta taxa de dados entre as ERB's e a CCC.

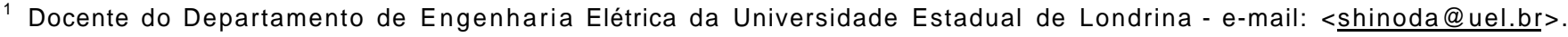


O simulador baseado no sistema descrito anteriormente, mede o grau de serviço para uma dada quantidade de canais nas ERB's. O simulador considera somente as estações móveis e as ERB's ignorando a configuração da CCC e da RTPC.

As características ou parâmetros das ERB's e das estações móveis são mantidas em estrutura de dados distintos. Enquanto a estrutura de dados das ERB's são mantidas na memória e a maioria dos mesmos são estáticos, a estrutura de dados das estações móveis trafegam entre os blocos funcionais do simulador.

Este modelo assume que a chegada das chamadas segue uma distribuição de Poisson. Característica dos usuários tais como as coordenadas $x$ e $y$, velocidade do movimento e duração da chamada são variáveis aleatórias geradas com uma distribuição uniforme apropriada. Assume-se que os usuários estçao se movimentando numa linha reta durante a duração da chamada.

A medida que as chamadas chegam, as mesmas requisitam um canal (frequência). Se a ERB em que o usuário esta mais próxima possui canais livres, o canal é alocado ao usuário caso contrário a chamada é bloqueada. A medida que o usuário se move, a qualidade do canal é periodicamente testado e caso a qualidade esteja num nível crítico, o canal é liberado e outra ERB, com uma qualidade de sinal aceitável, aloca um canal disponível para o usuário (handoff).

As chamadas saem do sistema quando ocorrem um dos três eventos:

- término da chamada;

- chamada está fora da área de cobertura do sistema;

- bloqueado.

\section{BONeS DESIGNER}

O simulador foi construido empregando um software para simulação de redes, BONeS DESIGNER versão 3.6 (BONeS).

BONeS é um pacote integrado de software para modelamento e simulação de sistemas a eventodiscreto. O software permite uma interface gráfica no modelamento e divisão das funções no sistema a ser simulado. Com isso é possível a construção do sistema hierarcamente e graficamente, selecionando os blocos funcionais necessários dentro da sua biblioteca, ou aqueles desenvolvidos pelo próprio usuário.

Pode-se especificar a funcionalidade dos módulos utilizando uma combinação de três métodos:

- Hierarquia do diagrama de bloco;

- Máquina de estados finito;

- Código $\mathrm{C} / \mathrm{C}++$.

Com cada um desses métodos, o BONeS executa uma simulação a evento-discreto de Monte-Carlo, calculando a performance e mostrando os resultados graficamente.

\section{RESULTADOS}

O simulador, construido no BONeS, consiste de funções para geração de usuários, seleção da melhor ERB para a chamada, alocação do canal, determinação da localização do usuário e o monitoramento da qualidade do canal empregado pela estação móvel em vários pontos durante a chamada (Figura 1).

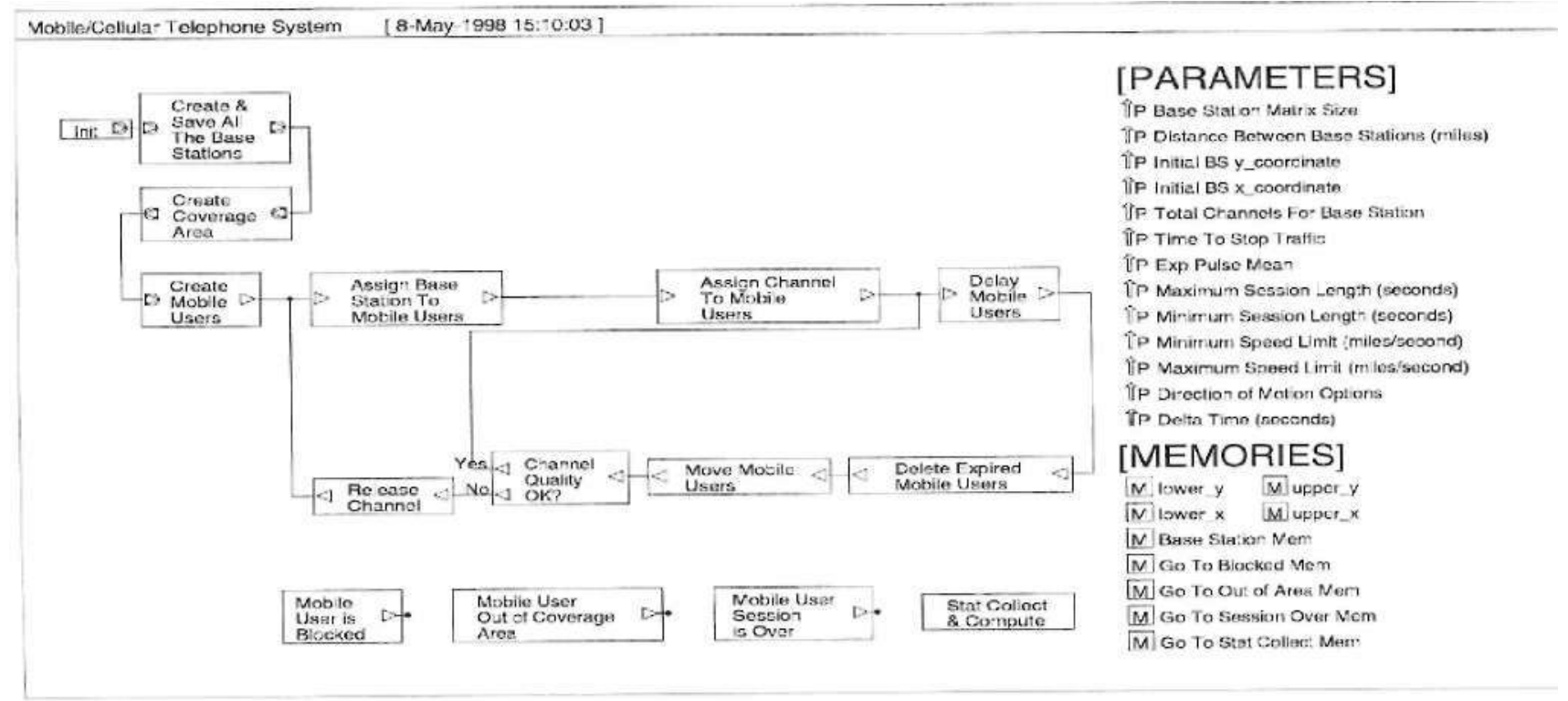

Figura 1 - Sistema Celular. 
O simulador inicia a avaliação do sistema através da determinação das ERB's e identificação das áreas de cobertura correspondentes. Isto é feito baseado nos parâmetros Base Station Matriz

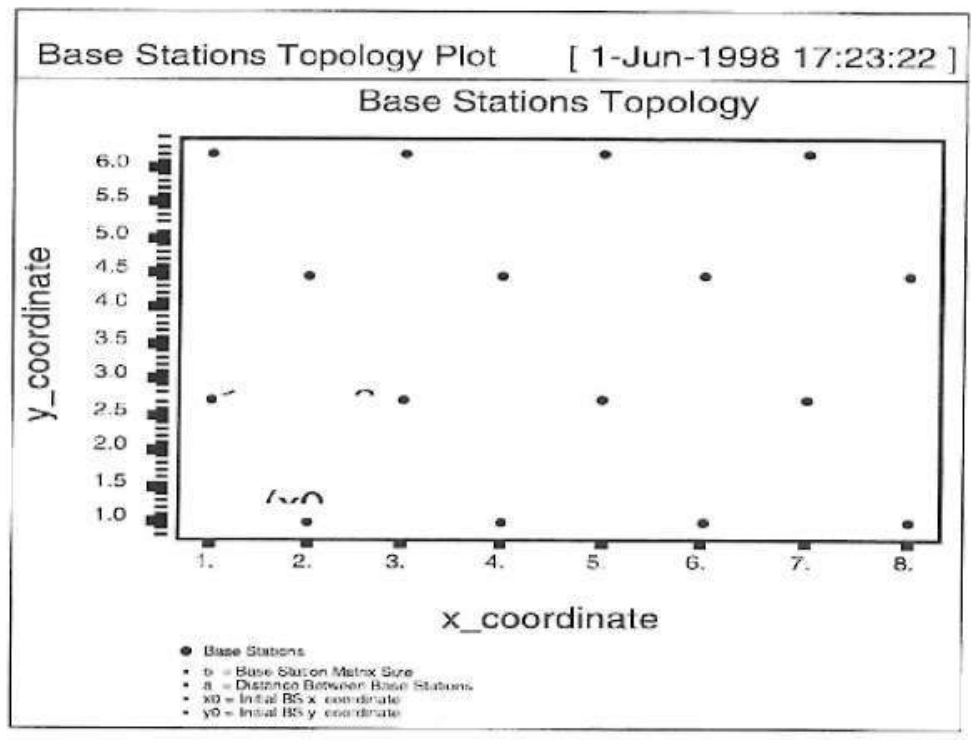

Figura 2 - Topologia das ERB's.

O sistema gera os usuários e o seus atributos de acordo com os parâmetros especificados. Após as chamadas serem criadas no Create Mobile Users bloco, as mesmas identificam a melhor ERB para o seu atendimento na Assign Base Station to Mobile Users bloco.

O próximo bloco é o Assign Channel to Mobile Users que seleciona um canal livre para a chamada. Caso não haja canal disponível, a chamada é bloqueada. O estado da chamada com o canal alocado é mantido inalterado durante um intervalo de tempo determinado pelo parâmetro Delta Time.

Após esse período de tempo no bloco Delay Mobile Users o Delete Expired Mobile Users bloco testa se houve término da chamada. Caso a chamada ainda esteja ativa, o próximo bloco é o Move Mobile Users para alterar o estado atual da chamada.

O Move Mobile Users bloco determina a localização mais recente (mudança de estado) da chamada e testa se o usuário esta dentro da área de cobertura. Chamadas fora da área de cobertura não são atendidas pelo sistema. A Figura 4 ilustra a movimentação da chamada na área de serviço.
Size, Distance Between Base Station (vide Figura 1) e as coordenadas $(x, y)$ da primeira ERB. A localização das ERB's e a área de cobertura total do sistema são mostradas na Figura 2 e Figura 3.

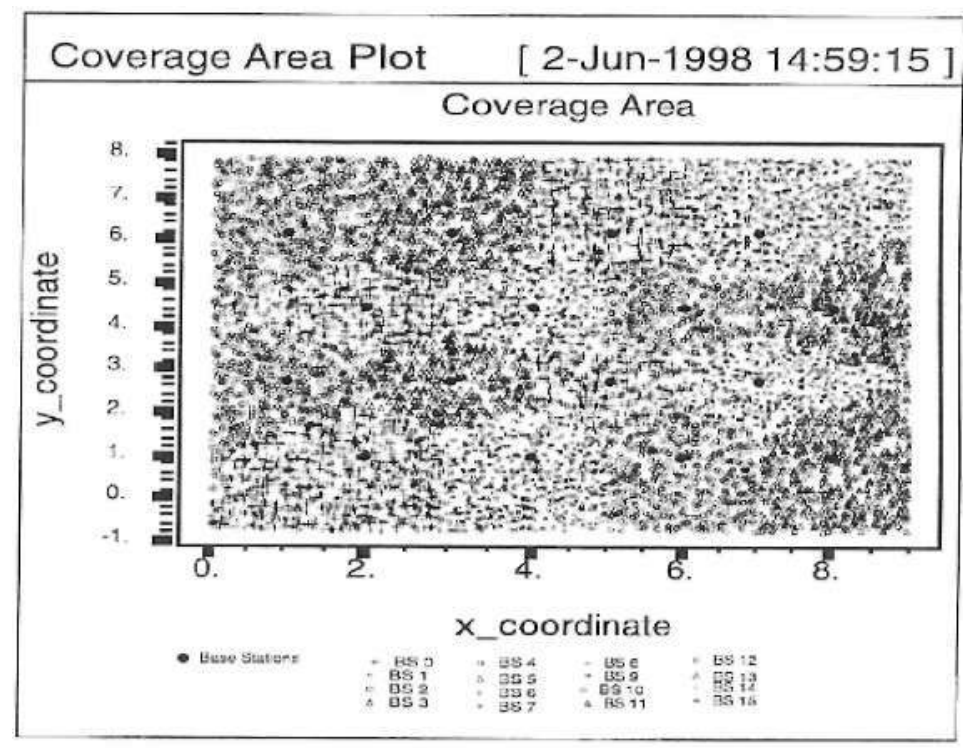

Figura 3 - Área de cobertura.

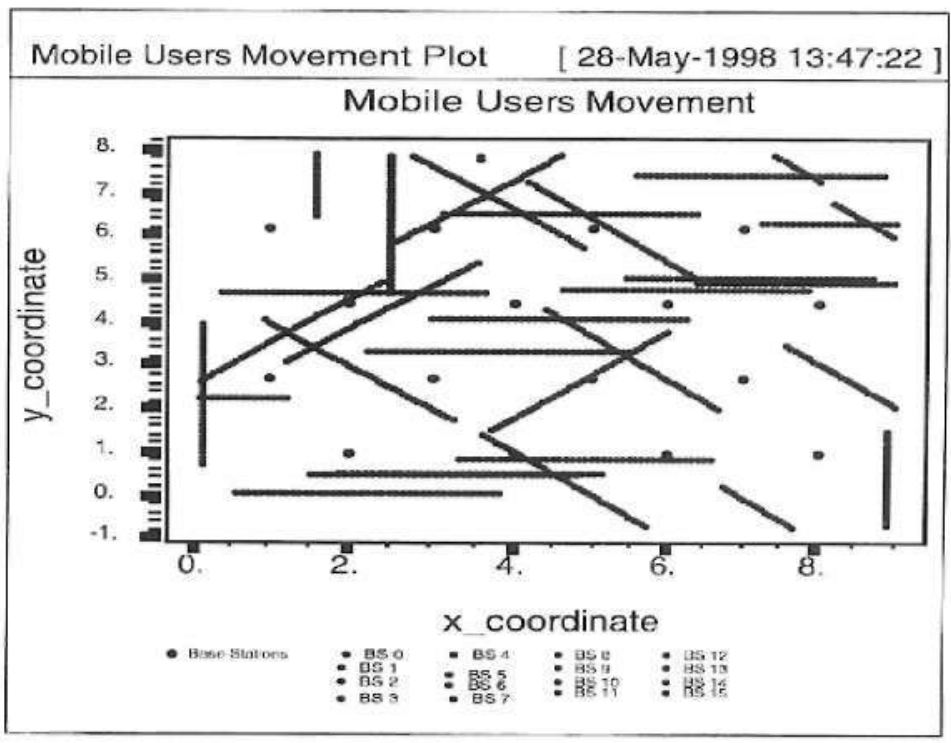

Figura 4 - Movimento do usuário/chamada.

O canal empregado pela chamada é testado no bloco Channel Quality OK. Se a qualidade do sinal estiver num nível razoável, a chamada volta novamente para o bloco Delay Mobile Users. Caso contrário, o canal é liberado (bloco Release Channel) com uma nova ERB e canal associada a chamada (vide Figura 1).

Os parâmetros utilizados na simulação estão descritos na Tabela 1 . 
Tabela 1 - Parâmetros empregado no simulador FDMA.

\begin{tabular}{|c|c|}
\hline Parâmetro & Valor \\
\hline Base Station Matriz Size & $4(16$ ERB's) \\
\hline Distance between Base Statior & 2 \\
\hline Initial BS y coordinate & 1 \\
\hline Initial BS x coordinate & 2 \\
\hline Total Channels for Base & $5,10,15,20,25$ \\
Station & \\
\hline Time to Stop Traffic & TSTOP \\
\hline Exp Pulse Mean & 1.0 \\
\hline Maximum Session Length & 240.0 \\
\hline Minimum Session Length & 30.0 \\
\hline Maximum Specd Limit & 70 \\
\hline Minimum Speed Limit & 20 \\
\hline Direction of Motion Options & 8 \\
\hline Delta Time & a/ $\mathrm{v} / 10$ \\
\hline TSTOP & 500 \\
\hline
\end{tabular}

Onde a é a distância entre ERB $(\mathrm{Km})$ e $v$ a velocidade máxima do usuário $(\mathrm{Km} / \mathrm{s})$.

Os resultados da simulação, com a configuração da Tabela 1 , estão nos gráficos da Figura 5 até Figura 8.

A Figura 5 indica a relação do grau de serviço em função da quantidade de canais por ERB. Para um grau de serviço de $95 \%$ são necessários 14 canais por ERB.

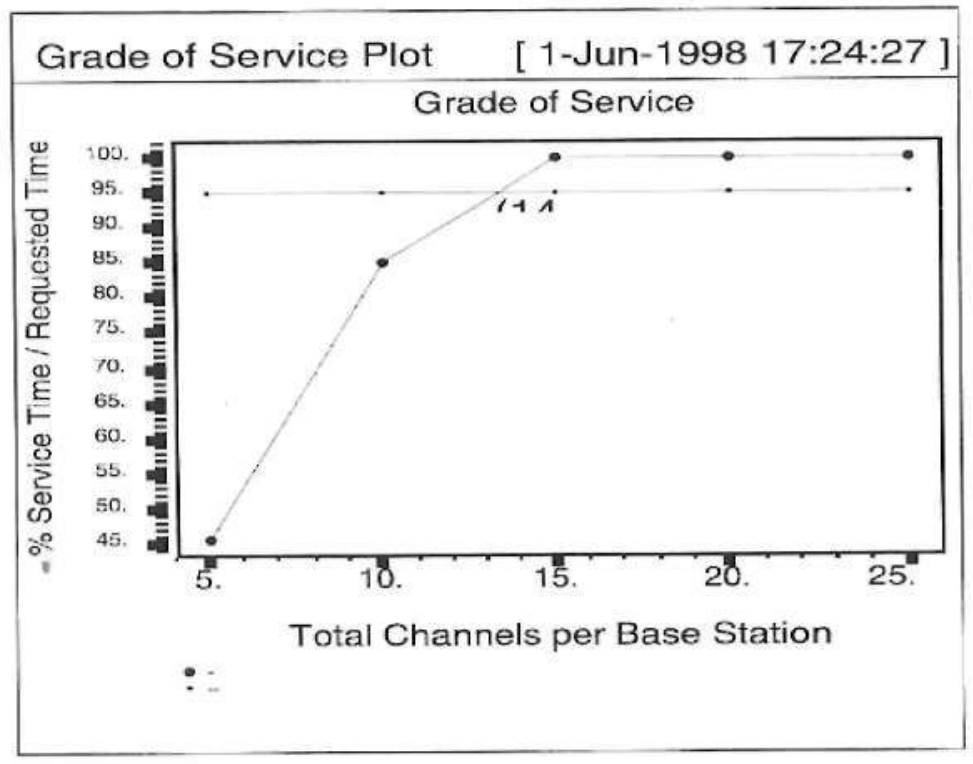

Figura 5 - Grau de serviço.

A Figura 6 indica a probabilidade de handoff durante a duração da chamada, tendo como parâmetro a quantidade de canais por ERB's. Observe que quanto menor a quantidade de canais por ERB's, maior a probabilidade de não ocorrer handoff por falta de canais nas células vizinhas.
Mobile Users Handoffs Distribution Plot ～[ 1-Jun-1998 17:24:27 ]

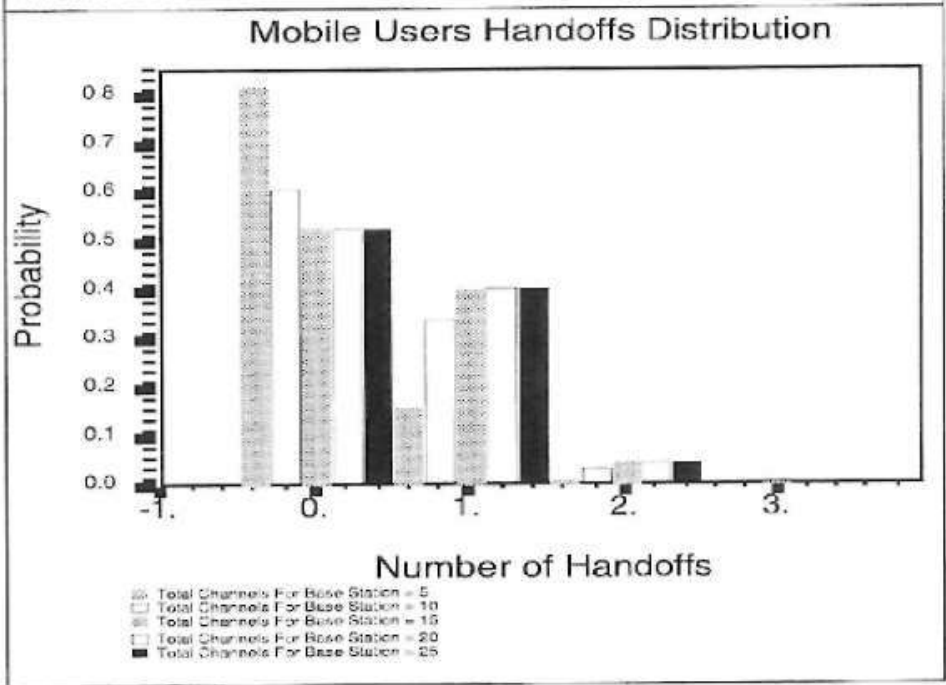

Figura 6 - Distribuição de Handoff.

A Figura 7 descreve a duração de chamadas de vários tipos. A duração está associada ao tempo médio da chamada e também da quantidade de canais disponíveis, no caso do handoff.

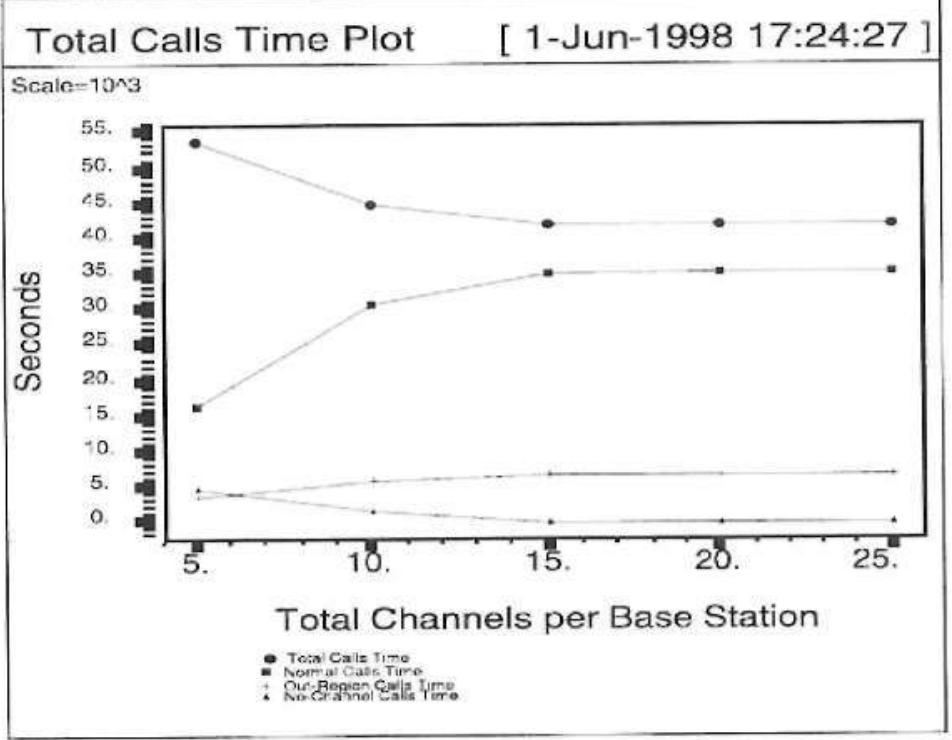

Figura 7 - Tempo total das chamadas.

A Figura 8 ilustra o número total de chamadas atendidas ou não por ERB durante a simulação. Note que quanto maior o número de canais maior o número de chamadas atendidas, mas há uma quantidade ótima de canais (no caso é 15) a partir do qual não ocorre uma melhora significativa do sistema. Este valor ótimo de canais depende do tráfego e do valor médio da duração da chamada. 


\section{Total Calls Count Plot [ 1-Jun-1998 17:24:27 ]}

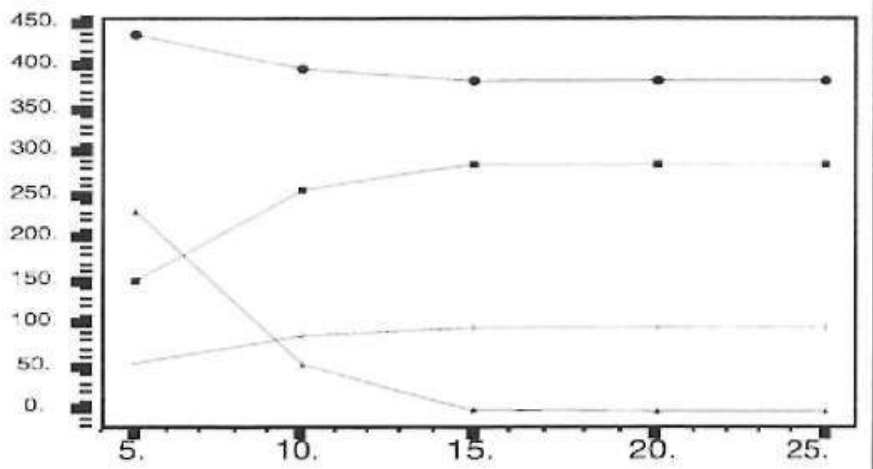

Total Channels per Base Station - Totú Cals Coun: :

Figura 8 - Número total de chamadas.

\section{CONCLUSÃO}

Este artigo descreveu o modelo e a impiementação de uma rede celular baseada em FDMA, construido através do BONeS. Além disso foram mostrados alguns resultados do simulador. O simulador pode ser útil como uma ferramenta de otimização de parâmetros do sistema TDMA, via implementação de alguns blocos adicionais. Um trabalho futuro poderia ser a construção de um simulador de um sistema celular CDMA (IS95), levando em consideração vários parâmetros no desempenho e otimização em termos de rede, sem entrar em detalhes na interface aérea.

SHINODA, A. A. Cellular Network Simulator based on FDMA system (AMPS/TDMA). Semina: Ci. Exatas/ Tecnol. Londrina, v. 21, n. 4, p. 47-51, dez. 2000.

ABSTRACT: This article describes the model and implementation of a cellular network simulator based on FDMA system (AMPS/TDMA). The purpose of the simulator is to evaluate the performance of the FDMA cellular network as well as the optimization of some parameters. Some results obtained from the simulator are also shown.

KEY WORDS: cellular network; simulation; FDMA; AMPS; TDMA.

\section{REFERÊNCIAS BIBLIOGRÁFICAS}

BONeS DESIGNER Alta Group of Cadence Design Systems Inc.

MAC DONALD, V. H. Advanced Mobile Phone Service. IEEE Trans. Veh. Tech., v. 49, p. 238-244, May 1980.
WOERNER, B. D. etal. Simulation Issues for Future Wireless Modems. IEEE Comm. Mag., p. 42-53, Jul. 1994.

TRANTER, W. H., KOSBAR, K.L. Simulation of Communication Systems. IEEE Comm. Mag., p. 26-35, Jul. 1994. 